\title{
NÍVEIS DE SÓDIO NA RAÇÃO DE FRANGAS DE 7 A 12 SEMANAS DE IDADE ${ }^{1}$
}

\author{
Sodium levels for pullets aged between 7 and 12 weeks
}

\author{
Marcelo Luís Gomes Ribeiro ${ }^{2}$, José Humberto Vilar da Silva², José Anchieta de Araújo ${ }^{3}$, \\ Paulo Borges Rodrigues ${ }^{4}$, Fernando Guilherme Perazzo Costa ${ }^{5}$, José Jordão Filho ${ }^{6}$
}

\begin{abstract}
RESUMO
O experimento foi realizado para estimar as exigências de sódio em frangas leves (LV) e semipesadas (SP), durante a fase de recria (7 a 12 semanas de idade). $O$ delineamento experimental foi inteiramente ao acaso, em esquema fatorial com seis níveis de sódio $(0,04 ; 0,10 ; 0,16 ; 0,21 ; 0,27$ e $0,32 \%)$ e duas linhagens (LV e SP), que resultaram em doze tratamentos, com cinco repetições de seis aves. Foram avaliados o consumo de ração (CR), consumo de água (CAG), ganho de peso diário (GPD), conversão alimentar (CA), temperatura retal (TR), comprimento da crista (CC), matéria seca das excretas (MSF) e umidade das excretas (UF). Não houve interação dos níveis de sódio (NS) com a linhagem sobre as variáveis estudadas. O CR não foi afetado pelos NS, porém as frangas SP consumiram mais ração que as LV. Os NS proporcionaram efeito quadrático para o GPD e CA, e a linhagem LV apresentou maior CC que a SP. O aumento do NS da ração elevou o CAG, a UF e diminuiu a MSF, mas a TR não foi alterada. Recomenda-se de 7 a 12 semanas de idade $0,22 \%$, ou o consumo de 123 e $140 \mathrm{mg}$ de sódio para as LV e SP, respectivamente.
\end{abstract}

Termos para indexação: Bicarbonato de sódio, desempenho, balanço eletrolítico, avicultura.

\begin{abstract}
The experiments were carried out to determine sodium requirements for light (LS) and egg-brown (EBS) pullets from 7 to 12 weeks of age. It was used a completely randomized design according to a $6 \times 2$ factorial (sodium levels: $0.04 ; 0.10 ; 0.16 ; 0.21 ; 0.27$ and $0.32 \%$ X 2 strains), with five replicates of six pullets per experimental unit. Feed intake, water intake, daily weight gain, feed: gain ratio, rectal temperature, and comb size were evaluated, as well as feces dry matter and humidity. In the two experiments, there was no interaction $(\mathrm{P}>0.05)$ between sodium levels $(\mathrm{SL})$ and strains on the studied variables. Feed intake was not affected by LS, but it was greater in EBS than in LS birds ( $\mathrm{P}<0.05)$. LS had significant effects on daily weight gain and feed: gain ratio according to the quadratic model, and comb size was greater in LS compared to EBS birds. The increase in dietary sodium levels increased water intake and feces humidity, whereas feces dry matter decreased. Rectal temperature was not affected by the treatments. For SL and EBS, it is recommended $0.22 \% \mathrm{Na}$ or a daily intake of 123 and $140 \mathrm{mg}$ of sodium, respectively, from 7 to 12 weeks of age.
\end{abstract}

Index terms: Sodium bicarbonate, performance, electrolyte balance, aviculture.

(Recebido em 29 de maio de 2007 e aprovado em 13 de dezembro de 2007)

\section{INTRODUÇÃo}

As exigências nutricionais e os programas de alimentação de poedeiras em crescimento não têm merecido a mesma atenção daqueles de poedeiras em produção. Por isso, os programas de alimentação, atualmente usados, precisam ser reavaliados, a fim de determinar se são biologicamente efetivos no atendimento das mudanças nas exigências de poedeiras jovens, em todos os estágios, durante o período de crescimento (SILVA, 2000).
É importante avaliar-se o comportamento das características genéticas relacionadas à produção de ovos das linhagens, em função de alterações nos níveis nutricionais das dietas, em virtude do principal motivo da criação de frangas de reposição que é obter delas o máximo desempenho durante o ciclo produtivo.

A alimentação é o principal item do custo de produção de aves e, por isto, a definição das especificações nutricionais das rações é a ferramenta mais eficiente para reduzir esse custo, desde a cria e recria de frangas até o primeiro e o segundo ciclos de postura.

\footnotetext{
${ }^{1}$ Parte da Tese de Doutorado do primeiro autor

2Doutores, Professores - Centro de Ciências Humanas, Sociais e Agrárias/CCHSA - Departamento de Agropecuária/DAP - Universidade Federal da Paraíba/UFPB - Cidade Universitária - Cx. P. 14 - 58220-000 - Bananeiras, PB - marcelolgr@hotmail.com; jvilar@cft.ufpb.br - PICDT/Capes ${ }^{3}$ Mestrando em Zootecnia - Centro de Ciências Agrárias/CCA - Departamento de Zootecnia/DZ - Universidade Federal da Paraíba/UFPB - Rodovia PB 079 - Km 12 - 58397-000 - Areias, PB - araujosertao@hotmail.com

${ }^{4}$ Doutor, Professor - Departamento de Zootecnia/DZO - Universidade Federal de Lavras - Cx. P. 3037 - $37200-000$ - Lavras, MG pbrodrigues@pesquisador.cnpq.br

${ }_{5}^{5}$ Doutor, Professor - Centro de Ciências Agrárias/CCA - Departamento de Zootecnia/DZ - Universidade Federal da Paraíba/UFPB - Rodovia PB 079 - Km 12 - 58397-000 - Areias, PB - fperazzo@cca.ufpb.br

${ }^{6}$ Mestre, Professor - Centro de Ciências Humanas, Sociais e Agrárias/CCHSA - Departamento de Agropecuária/DAP - Universidade Federal da Paraíba/UFPB - Cidade Universitária - Cx. P. 14 - 58220-000 - Bananeiras, PB - jjordaofilho@yahoo.com.br
} 
No Brasil, o clima quente e as altas densidades de alojamento afetam o bem-estar e deprimem o consumo alimentar das aves. Portanto, o equilíbrio da relação energianutriente, especialmente dos nutrientes que são adicionados em pequenas proporções nas rações, como os minerais e as vitaminas, é primordial para otimizar o desempenho das aves.

Os estudos desenvolvidos com minerais têm priorizado aqueles com mais alto nível de inclusão e os de maior impacto no custo final da ração. Por isso, o fósforo e o cálcio que são, por ordem, o terceiro e o quarto nutriente mais caro, tem atraído mais atenção que o sódio, cloro e potássio. Entretanto, minerais como o sódio, que têm baixo preço e alta disponibilidade, são tão importantes quanto os demais para o metabolismo animal. Silva et al. (2006) comentaram que o sódio é essencial para prevenir estados carenciais e, consequientemente, patologias nutricionais multifatoriais, em virtude de o sódio atuar na absorção de aminoácidos, carboidratos, água, minerais (cálcio) e vitaminas hidrossolúveis.

Além disso, a importância da manutenção das funções vitais do organismo está associada à presença desse cátion nos fluidos extracelulares, atuando no equilíbrio ácido-básico e na pressão osmótica, assim como na transmissão do impulso elétrico nas células nervosas, movimentos do músculo cardíaco e permeabilidade celular (GUYTON \& HALL, 1997).

O bicarbonato de sódio tem sido avaliado na nutrição de aves para estimar o consumo e água e diminuir o estresse calórico, particularmente, durante o verão (BORGES, 2006), na melhoria da qualidade da casca do ovo (FARIA et al., 2000; JUNQUEIRA et al., 2000, 2003) e estimar a exigência de sódio em aves (MURAKAMI, 2003; RIBEIRO et al., 2007).

O NRC (1994) recomenda $0,15 \%$ de sódio na dieta de 1 a 18 semanas de idade para frangas leves e as semipesadas. Leeson \& Summers (1997) sugeriram 0,18\% de sódio na dieta de frangas leves, enquanto Rostagno et al. (2005) sugeriram 0,16\% de sódio de 7 a 12 semanas, para as duas linhagens.

No Brasil, existem escassas informações sobre as exigências de sódio para frangas na fase de reposição. Portanto, objetivou-se com este trabalho estimar as exigências de sódio de frangas leves e semipesadas, de 7 a 12 semanas de idade.

\section{MATERIAL E MÉTODOS}

O experimento foi conduzido no Laboratório de Pesquisa em Nutrição de Aves (LPNA) do Centro de Formação de Tecnólogos (CFT-UFPB), Campus III, situado na cidade de Bananeiras-PB, micro-região do Brejo paraibano, de maio a setembro/2003, em galpão de alvenaria medindo $24 \mathrm{~m}$ de comprimento por $9 \mathrm{~m}$ de largura, pé direito de $2,80 \mathrm{~m}$, e orientação Leste-Oeste. As laterais do galpão foram protegidas com telas de arame e muretas de 0,40 m de altura. A cobertura, em duas águas de telha de barro, beirais de 1,50 m nas duas laterais e sem lanternim. Os boxes internos dispostos em duas fileiras, com piso coberto com cama de maravalha, medindo cada um 1,0 x 1,5 m. Cada boxe cercado com tela plástica, contendo um comedouro tubular infantil (capacidade de $4 \mathrm{~kg}$ de ração) e dois bebedouros tipo nipple, cada um com coletor plástico, para prevenir desperdício de água.

O experimento foi conduzido com 360 aves, sendo 180Lohmann-LSL e 180 Lohmann Brown(LB), selecionadas e padronizadas pelo peso vivo (LSL $451 \pm 5$ e LB 500 $\pm 5 \mathrm{~g}$ ), no período de 7 a 12 semanas de idade. $\mathrm{O}$ delineamento experimental foi inteiramente ao acaso, em esquema fatorial 6 x 2 (níveis de sódio X linhagem), totalizando 12 tratamentos, com cinco repetições de seis aves.

Os dados de temperatura e umidade relativa do ar foram registrados três vezes ao dia, utilizando-se um termohigrômetro digital, no interior de um boxe localizado no centro do galpão.

As rações foram formuladas à base de milho e farelo de soja, para atender às exigências nutricionais das aves em todos os nutrientes (NRC, 1994; ROSTAGNO et al., 2000), exceto em sódio, que foi suplementado pelo bicarbonato de sódio ( $27 \%$ de sódio), em substituição ao inerte (areia) nos valores de 0,$00 ; 0,20 ; 0,41 ; 0,61 ; 0,86$ e $1,15 \%$, resultando em seis níveis crescentes de sódio na ração $(0,04 ; 0,10 ; 0,16$; 0,$21 ; 0,27$ e $0,32 \%$ ) e balanços eletrolíticos de $157 ; 183 ; 209$; 231; 257; e $278 \mathrm{mEq} / \mathrm{kg}$. A ração basal, utilizada no ensaio, foi formulada para conter $2.900 \mathrm{kcal}$ de EMAn/ $\mathrm{kg}$ de ração e, $15,9 \%$ de proteína bruta. Os níveis de sódio e potássio calculados de $0,04 \%$ e $0,62 \%$ foram semelhantes aos analisados de 0,04 e 0,60 \% na ração referência (Tabela 1).

As variáveis estudadas foram consumo de ração, consumo de água, ganho de peso diário, conversão alimentar, matéria seca e umidade das excretas, comprimento de crista e a temperatura retal, de acordo com os níveis de sódio adicionados à ração.

O consumo de ração (g/ave/dia) foi calculado ao final de cada subperíodo de 14 dias pela diferença entre a ração fornecida e a sobra de ração e apresentado em valores médios, no período total avaliado (7 a 12 semanas). $\mathrm{O}$ ganho de peso diário foi o peso final, em cada subperíodo, menos o peso inicial, dividido pelo número de dias, enquanto a conversão alimentar foi o resultado da divisão do consumo de ração pelo ganho de peso.

O consumo de água (em temperatura ambiente) foi medido, semanalmente, pela diferença entre a quantidade 
de água fornecida e a sobra de água em cada balde por boxe, enquanto a umidade das excretas foi avaliada, no último de dia de cada subperíodo, pela coleta de material em bandejas de metal colocadas dentro dos boxes e, posteriormente, secas em estufa de circulação de ar forçada a $55^{\circ} \mathrm{C}$, durante 18 horas (SILVA, 1991).

Tabela 1 - Composições dos ingredientes e química da ração referência utilizada no experimento.

\begin{tabular}{|c|c|}
\hline Ingredientes & $\%$ \\
\hline Milho, $8,57 \%$ de PB e 3.371 kcal $\backslash$ EMAn ${ }^{2}$ & 70,31 \\
\hline Farelo de soja, $45 \%$ PB e 2.266 kcallEMAn $^{2}$ & 21,58 \\
\hline Calcário & 0,97 \\
\hline Fosfato bicálcico & 1,37 \\
\hline DL - Metionina & 0,08 \\
\hline Sal comum & 0,02 \\
\hline Cloreto de colina & 0,02 \\
\hline Premix vitamínico $^{3}$ & 0,08 \\
\hline Premix mineral $^{4}$ & 0,05 \\
\hline Anticoccidiano $^{5}$ & 0,04 \\
\hline Viginiamicina & 0,03 \\
\hline BHT $^{6}$ & 0,01 \\
\hline Cloreto de potássio & 0,21 \\
\hline Bicarbonato de sódio & variável \\
\hline Inerte (areia lavada) & 5,24 \\
\hline Total & 100,00 \\
\hline \multicolumn{2}{|l|}{ Composição calculada } \\
\hline Proteína Bruta, $\%^{7}$ & 15,70 \\
\hline Energia Metabolizável, (kcal/kg) & $2.900,00$ \\
\hline Cálcio (\%) & 0,80 \\
\hline Fósforo Disponível (\%) & 0,35 \\
\hline Metionina $(\%)$ & 0,34 \\
\hline Metionina+Cistina (\%) & 0,61 \\
\hline Lisina $(\%)$ & 0,78 \\
\hline Treonina (\%) & 0,62 \\
\hline Triptofano $(\%)$ & 0,18 \\
\hline Sódio $(\%)^{8}$ & 0,04 \\
\hline Potássio $(\%)^{8}$ & 0,71 \\
\hline Cloro (\%) & 0,15 \\
\hline
\end{tabular}

${ }^{1}$ Exigências nutricionais de acordo com as recomendações do NRC (1994) e Rostagno et al. (2000).

${ }^{2}$ Valores tabelados (ROSTAGNO et al., 2000).

${ }^{3}$ Premix vitamínico- kg do produto. Vit. A, 40.000.000 UI; Vit. D3, 8.000.000 UI; Vit. E, 100.000 UI; Vit. K , 6.000,0 mg; Vit. B, 6.000,0 mg; Vit. $\mathrm{B}_{2}, 20.000,0 \mathrm{mg}$; Vit. $\mathrm{B}_{6}, 12.000,0 \mathrm{mg}$; Vit.B ${ }_{12}, 60.000,0 \mathrm{mg}$; Biotina , 320,0 mg; Ácido fólico, $2.800 \mathrm{mg}$; Ácido Nicotínico, 120.000,0 mg; Ácido Pantotênico, 40.000,0 mg; Se, 1.000,0 mg.

${ }^{4}$ Premix mineral: kg do produto: Mn, $150.000 \mathrm{mg}$; Zn, $100.000 \mathrm{mg}$; Fe, $100.000 \mathrm{mg}$; Cu, $16.000 \mathrm{mg}$ e I, $1.500 \mathrm{mg}$.

${ }^{5}$ Salinomicina, ${ }^{6}$ Antioxidante. BHT(Butil-hidroxi-tolueno). Níveis de garantia do produto- BHT $100 \mathrm{~g} / \mathrm{kg}$.

${ }^{7}$ Valores analisados no Laboratório de Controle de Qualidade de Alimentos CFT\UFPB, pelo método de Kjeldahl.

${ }^{8}$ Valores analisados: $\mathrm{Na}=0,0405$ e K = 0,70; - Laboratório de Análise de Tecido de Planta - CCA-UFPB, seguindo metodologia descrita por Tedesco (1982). 
A temperatura retal foi medida com termômetro introduzido em $2 \mathrm{~cm}$ na cloaca das aves, por quatro minutos, em duas aves de cada parcela, no final de cada um dos três períodos de 14 dias.

No final da $12^{\mathrm{a}}$ semana, as aves foram colocadas em gaiolas separadas por tratamentos, recebendo ração única de recria II, e posteriormente foi medido o comprimento da crista na $15^{\mathrm{a}}, 16^{\mathrm{a}}$ e $17^{\mathrm{a}}$ semanas de idade, retirando-se a média, segundo método descrito por Keeling et al. (2003).

As análises estatísticas foram realizadas no programa SAEG, UFV (1982) e as médias dos tratamentos foram comparadas pelo teste $\mathrm{F}(\mathrm{P}<0,05)$. As diferenças entre as linhagens foram avaliadas pelo teste $\mathrm{SNK}(\mathrm{P}<0,05)$. As exigências de sódio foram estimadas pelo desdobramento das somas de quadrado de tratamento nos efeitos linear e quadrático, e o modelo foi escolhido pelo nível de significância, valor do coeficiente de determinação e resposta biológica das aves.

\section{RESULTADOS E DISCUSSÃO}

Durante o experimento, os dados médios de temperatura e umidade relativa do ar, máximas e mínimas, foram respectivamente, de 25 e $20 \mathrm{C}^{\mathrm{o}}$ e 98 e $78 \%$.

Não houve interação $(\mathrm{P}>0,05)$ dos níveis de sódio com a linhagem da ave sobre as variáveis estudadas e, somente os efeitos principais estão sendo apresentados (Tabela 2). Para o consumo de ração não houve diferença significativa em relação aos níveis de sódio, porém as frangas da linhagem semipesada consumiram mais 13,6\% de ração que as frangas leves $(\mathrm{P}<0,05)$. Isso corrobora o consumo previsto pelo NRC (1994) de $10 \%$ a mais de ração pelas aves semipesadas, em comparação com as leves. Segundo Bertechini (1997), as frangas semipesadas apresentam maior peso corporal e, por isso, ingerem maior quantidade de ração para a sua mantença que as leves.

Os níveis de sódio afetaram de forma quadrática $(\mathrm{P}<0,05)$, o ganho de peso diário e a conversão alimentar das frangas.

Pelo ganho de peso diário (Figura 1), a exigência de sódio estimada foi de $0,22 \%$, a mesma estimada para conversão alimentar (Figura 2). O valor estimado de 0,22\% é superior aquele de 0,15\% recomendado pelo NRC (1994), e aquele sugerido por Lesson \& Summer (1997) de 0,18\% de sódio.

Não apenas a deficiência de sódio deprimiu o desempenho das aves, mas também o seu excesso (Figuras 1 e 2). Segundo Underwood \& Suttle (1999), a ingestão contínua de dietas deficientes, ou com excesso de sódio altera a concentração desse mineral nos tecidos e fluidos corporais, causa lesões bioquímicas e afeta as funções fisiológicas conduzindo ao surgimento de desordens metabólicas nas aves. Como o sódio ajuda a manter o equilíbrio ácido básico (McDOWELL, 1992), o mesmo se relaciona com o metabolismo protéico, energético e mineral que afeta diretamente o desempenho das aves (PATIENCE, 1990). Scott et al. (1982), descreveram que os sintomas típicos de deficiência de sódio nas aves são a redução do crescimento, enfraquecimento ósseo e comprometimento das funções celulares.

Tabela 2 - Efeito dos níveis de sódio sobre o consumo de ração (CR), ganho de peso diário (GPD), conversão alimentar, (CA), consumo de água (CAG), matéria seca das excretas (MF), umidade das excretas (UF), temperatura retal (TR) e comprimento da crista (CC) para frangas leves (LV) e semipesadas (SP) na fase de 7 a 12 semanas de idade.

\begin{tabular}{|c|c|c|c|c|c|c|c|c|}
\hline $\begin{array}{c}\text { Níveis de } \mathrm{Na} \\
(\%)\end{array}$ & $\begin{array}{c}\text { CR } \\
(\mathrm{g} / \mathrm{a} / \mathrm{d})\end{array}$ & $\begin{array}{l}\text { GPD }^{2,3} \\
(\mathrm{~g} / \mathrm{a} / \mathrm{d})\end{array}$ & $\begin{array}{l}\mathrm{CA}^{2,3} \\
(\mathrm{~g} / \mathrm{g})\end{array}$ & $\begin{array}{c}\mathrm{CAG}^{1} \\
(\mathrm{~mL} / \mathrm{a} / \mathrm{d})\end{array}$ & $\begin{array}{c}M F^{l} \\
(\%)\end{array}$ & $\begin{array}{l}U F^{l} \\
(\%)\end{array}$ & $\begin{array}{l}\mathrm{TR} \\
\left({ }^{\circ} \mathrm{C}\right)\end{array}$ & $\begin{array}{l}\mathrm{CC} \\
(\mathrm{cm})\end{array}$ \\
\hline 0,04 & 57,9 & 11,3 & 5,12 & 70,0 & 29,7 & 70,3 & 41,7 & 3,5 \\
\hline 0,10 & 61,5 & 14,1 & 4,36 & 72,6 & 27,0 & 73,0 & 41,7 & 3,6 \\
\hline 0,16 & 58,9 & 13,7 & 4,30 & 76,6 & 25,8 & 74,3 & 41,8 & 3,5 \\
\hline 0,21 & 58,8 & 14,1 & 4,17 & 77,8 & 25,3 & 74,7 & 41,7 & 3,6 \\
\hline 0,27 & 60,6 & 13,8 & 4,39 & 79,9 & 24,4 & 75,6 & 41,7 & 3,7 \\
\hline 0,32 & 60,6 & 13,7 & 4,42 & 86,1 & 21,3 & 78,7 & 41,8 & 3,6 \\
\hline $\mathrm{LV}$ & $55,9^{\mathrm{B}}$ & $12,8^{\mathrm{B}}$ & 4,37 & 75,6 & 24,9 & 75,1 & 41,7 & $3,8^{\mathrm{A}}$ \\
\hline SP & $63,5^{\mathrm{A}}$ & $14,1^{\mathrm{A}}$ & 4,50 & 78,7 & 26,2 & 73,8 & 41,7 & $3,2^{\mathrm{B}}$ \\
\hline $\mathrm{CV} \%$ & 5,69 & 6,47 & 8,33 & 8,05 & 12,8 & 4,38 & 0,20 & 3,34 \\
\hline
\end{tabular}

A,BMédias de cada variável seguida de letras distintas diferem pelo teste $\mathrm{F}(\mathrm{P}<0,05)$.

${ }^{1}$ Efeito linear. ${ }^{2} \mathrm{LRP},{ }^{3}$ Efeito quadrático. $\mathrm{P}<0,05$. 
$y=10,2817+36,4568 X-82,7260 X^{2} ;\left(R^{2}=0,76\right)$

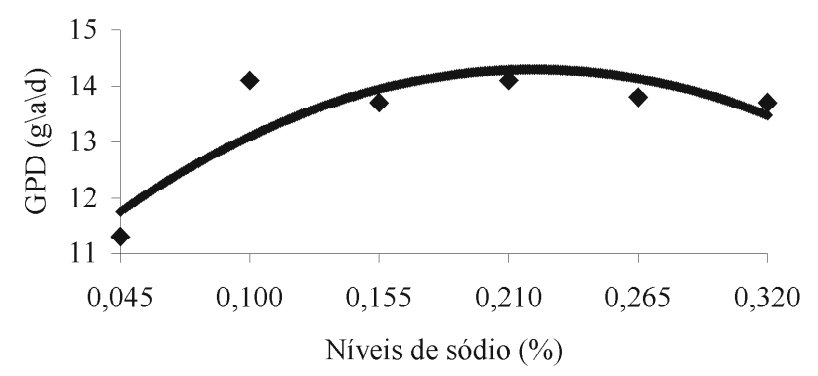

Figura 1 - Estimativas de exigência de sódio para ganho de peso diário (GPD), para frangas de 7 a 12 semanas de idade.

$$
y=5,68438-13,8437 X+32,1646 X^{2} ;\left(R^{2}=0,86\right)
$$

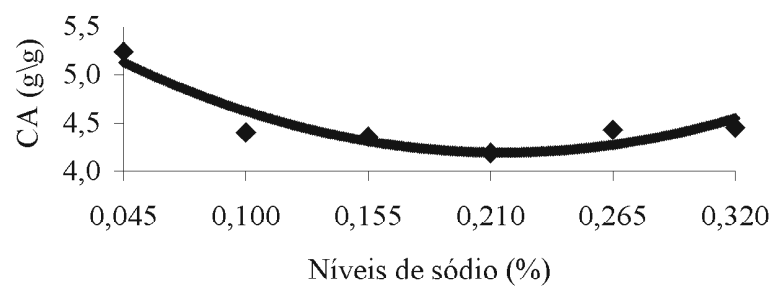

Figura 2 - Estimativas de exigência de sódio para conversão alimentar para frangas de 7 a 12 semanas de idade.

Por outro lado o excesso de sódio com a adição de bicarbonato de sódio à água de bebida tem contribuído para o surgimento de distúrbios patológicos em aves. Murabak \& Sharkawy (1999) trabalhando com níveis crescentes de bicarbonato de sódio $(0,0 ; 0,75 ; 2,0$ e 4,0 $\%)$ na água de bebida de frangas ( 3 semanas de idade), durante 35 dias, constataram que os grupos que receberam níveis de 0,75 a 2,0 \% de bicarbonato de sódio apresentaram quadro de gota visceral, e que a precipitação e cristalização de uratos nos rins das aves, estavam provavelmente relacionados às mudanças na concentração de eletrólitos, transtornos na pressão osmótica e alteração no pH do fluido extracelular, causados pelo excesso de bicarbonato de sódio ingerido. Barros et al. (2001) sugeriram que a piora no desempenho das aves com o excesso de sódio na ração, estaria possivelmente relacionada com o aumento do gasto energético através da bomba de sódio e potássio, no controle do gradiente eletrolítico entre o meio extra e intracelular ou, à toxidez causada pelo excesso de sódio na dieta.

Os níveis crescentes de sódio proporcionaram aumento linear no consumo de água e umidade das excretas (Tabela 2 e Figuras 3 e 4), enquanto a matéria das excretas (Figura 5) diminuiu de forma linear $(\mathrm{P}<0,05)$. Para Guyton \& Hall (1997), o equilíbrio de sódio no organismo envolve a produção de hormônios que estimulam a sua excreção ou retenção. Quando há excesso de água no corpo e a osmolaridade do líquido extracelular é reduzida, a secreção do hormônio antidiurético $(\mathrm{ADH})$ pela hipófise posterior é diminuída, reduzindo assim a permeabilidade dos túbulos renais, o que causa excreção de grande quantidade de urina.

Segundo Macari (1996), a adição de sais à ração (sódio, potássio, etc) provoca sensação de sede nas aves e, conseqüentemente estimula o consumo de água. No entanto, do ponto de vista fisiológico, não existirá quebra de homeostase, pois todo o excesso de água ingerido será excretado pelos rins ou excretas, produzindo excretas moles e cama molhada, o que pode prejudicar a saúde das aves. Os resultados do presente trabalho concordam com aqueles de Wideman \& Buss (1985), que constataram aumento no consumo de água e, conseqüentemente, da umidade das excretas, quando as aves consumiram rações com elevados níveis de sódio. Para Paiva (1998), esse aumento da umidade das excretas pode representar um grande problema, uma vez que essa situação contribui para o desenvolvimento de larvas e proliferação de moscas, nas instalações e áreas circunvizinhas da granja.

As aves leves apresentaram maior tamanho de crista que as semipesadas $(\mathrm{P}<0,05)$. O maior tamanho de crista das aves leves deve estar associado à influência da raça Legorne que, tradicionalmente, apresenta maior

$$
y=67,3466+53,7816 X ;\left(R^{2}=0,96\right)
$$

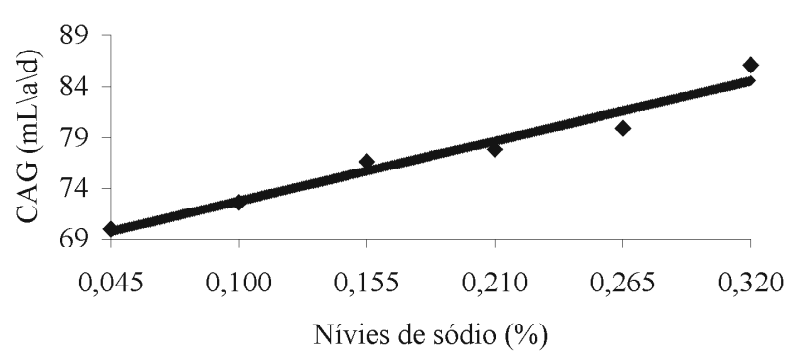

Figura 3 - Efeito linear dos níveis de sódio na ração sobre o consumo de água (CAG), em frangas de 7 a 12 semanas de idade. 


$$
y=69,6764+26,0164 X ;\left(R^{2}=0,93\right)
$$

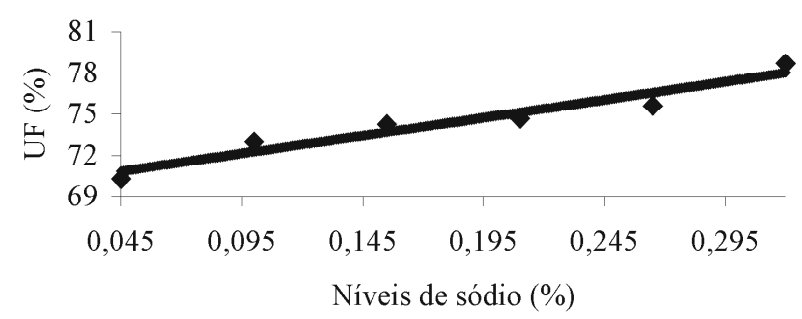

Figura 4 - Efeito linear dos níveis de sódio na ração sobre umidade das excretas, em frangas de 7 a 12 semanas de idade.

$$
y=30,3236-26,0164 X ;\left(R^{2}=0,93\right)
$$

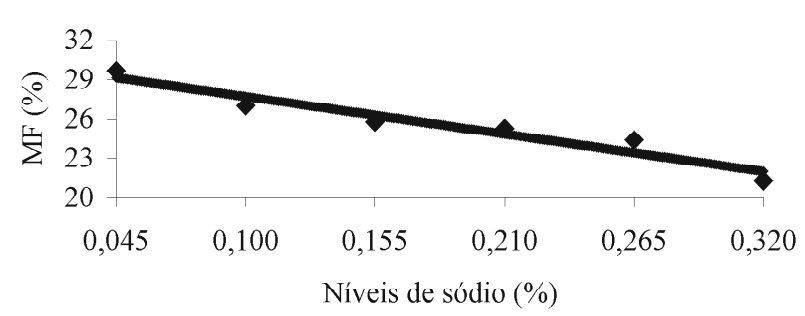

Figura 5 - Efeito linear dos níveis de sódio na ração sobre matéria seca das excretas, em frangas de 7 a 12 semanas de idade.

tamanho de crista que as aves da raça Rhode Island Red, que deram origem, respectivamente, às linhagens leves e semipesadas.

Apesar do maior consumo de água, com o aumento do nível de sódio através da adição de bicarbonato de sódio na ração, a temperatura retal das aves não foi alterada pelos tratamentos, possivelmente, porque as aves não estavam na condição de estresse pelo calor. O maior consumo de água pode ser explicado pela elevação do bicarbonato de sódio na ração. Segundo Furlan (2002), o bicarbonato de sódio tem sido um dos sais usados para aumentar o consumo de água, e aliviar os efeitos negativos das altas temperaturas. Junqueira et al. (1999) quando avaliaram a adição de bicarbonato de sódio e a associação desse com o cloreto de amônia $\left(\mathrm{NH}_{4} \mathrm{Cl}\right)$ na água de bebida para frangos de corte de 22 a 42 dias de idade, observaram que as aves expostas ao estresse agudo pelo calor, recebendo água suplementada com bicarbonato de sódio, apresentaram menores temperaturas corporais.

A exigência média de sódio, estimada pela regressão quadrática para a fase de 7 a 12 semanas de idade das aves considerando o ganho de peso diário e a conversão alimentar foi de $0,22 \%$, resultando em balanço eletrolítico da dieta de $235 \mathrm{mEq} / \mathrm{kg}$; valor superior aos $160 \mathrm{mEq} / \mathrm{kg}$ calculados por Rostagno et al. (2005).

\section{CONCLUSÕES}

Recomenda-se $0,22 \%$ de sódio para as aves leves e semipesadas de 7 a 12 semanas de idade, ou consumo diário de 123 e $140 \mathrm{mg}$ de sódio, respectivamente.

\section{REFERÊNCIAS BIBLIOGRÁFICAS}

BARROS, J. M. S.; GOMES, P. C.; ROSTAGNO, H. S. Exigência nutricional de sódio para frangos de corte de 1 a 21 dias de idade. Revista Brasileira de Zootecnia, Viçosa, v. 30, n. 3, p. 1044-1051, 2001.

BERTECHINI, A. G. Nutrição de monogástricos. Lavras: UFLA-FAEPE, 1997.

BORGES, S. B. Aplicação do conceito de balanço eletrolítico para aves. In: CONFERÊNCIA APINCO DE CIÊNCIA E TECNOLOGIA AVÍCOLA, FACTA, 2006, Santos, SP. Anais... Santos: FACTA, 2006. p. 123-137.

FARIA, D. E.; JUNQUEIRA, O. M.; SAKOMURA, N. K. Efeito de diferentes níveis de sódio e fósforo sobre o desempenho e a qualidade da casca dos ovos de poedeiras comerciais. Revista Brasileira de Zootecnia, Viçosa, v. 29, n. 2, p. 458-466, 2000.

FURLAN, R. L. et al. Equilíbrio ácido-básico. In: MACARI, M.; FURLAN, R. L.; GONZALES, E. Fisiologia aviária aplicada a frangos de corte. 2. ed. Jaboticabal: FUNEP, 2002. cap. 4, p. 51-76, 375 p.

GUYTON, A. C.; HALL, J. E. Tratado de fisiologia médica. Rio de Janeiro: Guanabara, 1997. 1014 p.

JUNQUEIRA, O. M.; ANDREOTTI, M. O.; RODRIGUES, E. A. Fontes e níveis de sódio em rações de poedeiras comerciais.. Acta Scientiarum Animal Sciences, Maringá, v. 25, n. 1, p. 79-84, 2003.

JUNQUEIRA, O. M.; CAMARGO FILHO, B.; ARAÚJO, L. F. Efeito das fontes e níveis de sódio, cloro e potássio e da relação $(\mathrm{Na}+\mathrm{K}) / \mathrm{Cl}$, sobre o desempenho e características do plasma sangüíneo de poedeiras comerciais. Revista Brasileira de Zootecnia, Viçosa, v. 29, n. 4, p. 1110-1116, 2000. 
JUNQUEIRA, O. M.; FONSECA, L. E. C.; ARAÚJO, L. F.; ARAÚJO, C. S. S.; SAKOMURA, N. K. Desempenho e parâmetros hematológicos de frangos de corte submetidos à restrição alimentar recebendo soluções hidroelétricas. Revista Brasileira de Ciência Avícola, São Paulo, v. 1, n. 1 p. 55-59, 1999.

KEELING, L. J.; ESTEVEZ, I.; NEWBERY, R. C. Productionrelated traits of layers reared in different sized flocks: the concept of problematic intermediate group sizes. Poultry Science, Champaign, v. 82, n. 9, p. 1393-1396, 2003.

LEESON, S.; SUMMERS, J. D. Commercial and poultry nutrition. Guelph: University Books, 1997. 350 p.

MACARI, M. Água na agricultura industrial. Jaboticabal: Unesp-Funep, 1996. 128 p.

McDOWELL, L. R. Minerals in animal and human nutrition. London: Academic, 1992. 522 p.

MURABAK, M.; SHARKAWY, A. A. Toxopathology of gout induced in laying pullets sodium bicarbonate toxicity. Environmental Toxicology and Pharmacology, [S.1.], v. 7, p. 227-236, 1999.

MURAKAMI, A. E.; FIGUEIREDO, D. F.; PERUZZI, A. Z. Níveis de sódio para poedeiras comerciais no primeiro e segundo ciclos de produção. Revista Brasileira de Zootecnia, Viçosa, v. 32, n. 6, p. 1674-1680, 2003.

NATIONAL RESEARCH COUNCIL. Nutrient requirements of poultry. 9. ed. Washington, DC: National Academy, 1994. 155 p.

PAIVA, D. P. Controle integrado de moscas em avicultura intensiva de postura: I. controle da criação de moscas no esterco. Concórdia: Embrapa Suíno e Aves, 1998.

PATIENCE, J. F. A review of the role of acid-base balance in amino acid nutrition. Journal of Animal Science, Champaign, v. 68, n. 2, p. 398-408, 1990.

RIBEIRO, M. L. G.; SILVA, J. H. V.; ARRUDA, A. M. V. Níveis de sódio na ração de frangas de reposição de 12 a
18 semanas de idade. Revista Caatinga, Mossoró, v. 20, n. 3, p. 49-56, jul./set. 2007.

ROSTAGNO, H. S.; ALBINO, L. F. T.; DONZELE, J. L. Tabelas brasileiras para aves e suínos: composição de alimentos e exigências nutricionais. Viçosa: UFV, 2000. $141 \mathrm{p}$.

ROSTAGNO, H. S.; ALBINO, L. F. T.; DONZELE, J. L. Tabelas brasileiras para aves e suínos: composição de alimentos e exigências nutricionais. Viçosa: UFV, 2005. $186 \mathrm{p}$.

SCOTT, M. L.; NESHEIM, M. C.; YOUNG, R. J. Essential inorganic elements: nutrition of the chicken. 3. ed. New York: ML Scott, 1982.

SILVA, D. J. Análises de alimentos: métodos químicos e biológicos. 2. ed. Viçosa: UFV, 1991. 160 p.

SILVA, J. H. V. Exigências nutricionais de lisina para frangas de postura, leves e semipesadas, nas fases de inicial, cria e recria. 2000. $149 \mathrm{f}$. Tese (Doutorado em Zootecnia) - Universidade Federal de Viçosa, Viçosa, 2000.

SILVA, J. H. V.; RIBEIRO, M. L. G.; JORDÃO FILHO, J. O Sódio afeta o crescimento de órgãos dos sistemas circulatório, digestivo e imune de frangas. Revista Ave World, [S.1.], n. 23, ago./set. 2006.

TEDESCO, M. J. Extração simultânea de N, P, K, Ca e Mg em tecido de plantas por digestão com H2O2- H2SO4. Porto Alegre: UFRGS, 1982. 23 p.

UNDERWOOD, E. J.; SUTTLE, N. F. General introduction. In: . Mineral nutrition of Livestock. Wageningen: CABI, 1999. p. 1-16.

UNIVERSIDADE FEDERAL DE VIÇOSA. SAEG -Sistema para análise estatística e genética. Viçosa, 1982. 59 p.

WIDEMAN, R. F.; BUSS, E. G. Arterial blood gas, pH and bicarbonate values in laying hens selected for thick or thin eggshell production. Poultry Science, Champaign, v. 64, n. 5, p. 1015-1019, 1985 